\title{
Notas sobre ecomunitarismo, educación ambiental y pensamiento indígena latinoamericano: los xavante y el Popol Vuh
}

\author{
Dr. Sirio Lopez Velasco*
}

\section{Resumen:}

En estas notas pretendemos dar un primer paso en el diálogo intercultural de nuestra propuesta ecomunitarista y la visión de educación ambiental que ella incluye, con el pensamiento indígena latinoamericano, en especial con la cultura xavante (que habita en Brasil) y la resumida en esa obra maestra maya que es el Popol Vuh. Para tanto destacamos algunas revelaciones, convergencias y divergencias entre ambos horizontes.

Palabras-clave: ecomunitarismo, educación ambiental, xavante, Popol Vuh

\section{Abstract:}

In this paper we introduce a intercultural dialogue between our ecommunitarianist theory, including his environmental education vision, and the LatinAmerican indigenous thought, especially the xavante culture (who lives in Brazil) and the maya's Popol Vuh. To do so, we emphasize some revelations, some convergences and some differences.

Key-words: ecommunitarianism, environmental education, xavante, Popol Vuh

\footnotetext{
Dr.enFilosofía.UniversidadFederaldeRioGrandeDoSul(FURG),Brasil.dlamji@hotmail.com
} 


\section{Introducción y advertencia}

Como trataremos de una tradición oral en lengua xavante, no hay más remedio que recordar el adagio que reza 'tradutore traditore' (el traductor es un traidor). Ahora bien, en nuestra defensa invocamos los siguientes hechos. En relación a los mitos xavantes, a) la traducción que usamos fue elaborada y revisada por xavantes bilingües (xavante-portugués), a partir de relatos de ancianos de la tribu, b) la obra que usamos fue elaborada por especialistas que convivieron largamente con la tribu xavante, y, c) abordaremos sólo las bases mínimas de los mitos xavantes. En relación al Popol Vuh: a) abordaremos sólo las bases mínimas de esa obra, y, b) lo haremos a la luz de una versión elaborada por un especialista que conoce las mejores versiones preparadas antes en diversas lenguas. Así esperamos evitar errores garrafales en la interpretación de las culturas que abordaremos aquí.

Dicho esto, aclaro que en estas líneas me propongo abordar una nueva fase de mi reflexión ecomunitarista, examinando lo que cada uno pensaría-viviría individual y/o colectivamente cuando, una vez superado el capitalismo (o sea, una vez dejada para atrás la "prehistoria humana", como dijo Marx), llegaría el momento de la verdadera historia propiamente humana, en la que serían abordadas las cuestiones cruciales de la vida y la muerte humanas y su relación con el resto de la naturaleza. En este trabajo pondré en relación el ecomunitarismo y educación ambiental ecomunitarista con los mitos y algunos aspectos básicos de la cultura xavante y del Popol Vuh. Aclaro que abordo los mitos xavantes y del Popol Vuh sin ningún instrumental antropológico especializado (por ejemplo del tipo estructural elaborado por Levy Strauss para interpretar mitos indígenas); simplemente comparo sus contenidos con mi propuesta ecomunitarista, intentando aprender de las culturas xavante y maya, a la vez que marco mis diferencias. 


\section{El ecomunitarismo: hacia el reino de la libertad}

Hemos dicho que el ecomunitarismo (ver, entre otros, Lopez Velasco 2003b y 2003c), aplicando las tres normas fundamentales de la ética (ver Lopez Velasco 2003a, 2003c, 2007 y 2008) se compondría de: a) una economía solidaria y ecológica, que basada en libres decisiones consensuales combine el principio "de cada uno según su capacidad y a cada uno según su necesidad" con la preservación-regeneración de una sana naturaleza (humana y no humana); hemos aclarado que esas 'necesidades' se pautarían en cada momento histórico por las tres normas fundamentales de la ética; b) una educación ambiental problematizadora que ayuda a ejercer la libertad individual de decisión (amparada por la primer norma de la ética) y la construcción consensual de respuestas a los problemas de la convivencia (exigencia de la segunda norma de la ética), c) una erótica del placer compartido que, haciendo parte de esa educación problematizadora y apoyada en sus frutos, supere el machismo, el sexismo, y la condena de la homosexualidad y de la masturbación, $y, d$ ) una "política de todos" que priorice la democracia directa (factible en grandes sociedades mediante los recursos de Internet) y donde las responsabilidades que sean indispensables se ejerzan rotativamente (ver Lopez Velasco 2003b, 2003c, 2007 y 2008).

Sobre esas bases habría llegado el momento en que cada ser humano acceda al tiempo y la tranquilidad necesarios para pensar-vivir los enigmas esenciales de la vida y de la muerte. Adelantándonos a la historia, abordamos desde ya un diálogo inicial entre ecomunitarismo y cultura indígena latinoamericana.

\section{El ecomunitarismo y los mitos y algunos aspectos de la cultura xavante}

Abordaremos nuestro tema en base a dos magníficos libros elaborados por B. Giaccaria y A. Heide; en uno de ellos son presentados (traducidos al portugués por un indígena controlado por el narrador) 25 mitos xavantes relatados por uno de los hombres más viejos de la tribu ("Jerônimo xavante conta"); en el otro los autores se limitan a presentar (sin interpretarlos) los rasgos fundamentales de la cultura xavante, observada con rigor y detalles ( "Xavante, povo autêntico"). Identificaremos las referencias de los asuntos tratados por el número romano del mito en el primer libro, o por las iniciales XPA seguidas de la página, para el segundo libro. 
De 1784 a 1860 lo xavantes vivieron en aldeas donde su expresión cultural propia fue hostilizada. Sólo después que lograron atravesar el Rio Araguaia y el Rio das Mortes, para establecerse en la Sierra do Roncador (en el contro-oeste del Brasil), la cultura xavante pudo retomar sus tradiciones con más autonomia. En 1946 los blancos volvieron a hacer contacto con la tribu y hubo divisiones y luchas intestinas, enfermedades y muerte de los más viejos. En los años 80 se verifica un reflorecimiento de la cultura xavante en casi todas las aldeas (26 en total). Uno de los suyos (Mário Juruna) llegó a ser Diputado Federal. En 1975 la población total xavante era de 4.500 individuos.

Como otros, los mitos xavante "explican" el origen de ciertas cosas y costumbres. Así se nos refiere el origen del oso hormiguero (I), del jaguar (I, "onça" en Brasil), del yacaré (VIII), del gavilán de cola blanca (XI), y del "jabuti" (reptil del orden de los quelonios, XX). La "explicación" del origen del fuego "explica" a su vez una de las fiestas rituales más importantes de los xavantes, la carrera del burití (en la que, por equipos, se van pasando un pedazo cilíndrico pesado de la palmera "burití", hasta llegar a la meta); se dice (I) que tal carrera fue inaugurada cuando por la primera vez los xavante conocieron el fuego robándoselo al "viejo jaguar" (que hasta entonces tenía figura humana y cobró su actual forma después de perder el monopolio del fuego) y tuvieron que transportarlo, mediante relevos, hasta la aldea; notemos que esta figura prometeica del origen del uso del fuego, se completa en el mismo mito con la aclaración de que también con el viejo jaguar los xavantes aprendieron a usar palitos para hacer fuego.

Hay dos mitos que también explican el origen de la lengua y los cantos xavantes (XVII y XXI).

También se refiere el origen del incesto entre madre e hijo $(X)$ y su prohibición, incluyendo explícitamente el caso de sobrino y tía (X); como la madre y el hijo que cometieron incesto se transformaron en "antas" (tapires) y fueron muertos en la caza por el padre, ello explica el origen del tabú alimentario de comer tapir de la mujer recién casada, hasta que tenga dos hijos; y los jóvenes iniciados en la ceremonia del WAY'A tampoco pueden comerlo.

Sobre la muerte, tres mitos refieren que antes los xavantes no morían (XXIV), que el primer animal que murió fue la lechuza (XXIII), y que los muertos van a las estrellas (II); otros explican como causa de muerte la enfermedad causada por el hecho de pasar cerca de las sepulturas (XXIV); y se nos dice que los muertos son enterrados (XII, quizá para que operen como "semillas" favorables a la agricultura (XPA, 84), y que esa costumbre también se extiende a los animales queridos (XXIII). 


\subsection{Revelaciones, convergencias y diferencias}

\subsubsection{Revelaciones}

Como en otras culturas mal denominadas "primitivas", Ilama la atención la alta cohesión social basada en la solidaridad que caracteriza a la cultura xavante. Así, para evitar que sólo el mejor cazador se beneficie con su destreza fuera de lo común, para todos los cazadores rige un código que establece con rigor qué parte de lo cazado (y de cada pieza) debe distribuirse a los parientes y al grupo; de forma que toda la comunidad (hombres y mujeres, viejos y niños, reciben lo necesario para bien comer); nótese que la administración de la comida (caza y agricultura) es dada a la mujer. También impera un código de regalos recíprocos que establece que todos deben dar y recibir, pero nadie puede pedir más de lo que el otro puede dar, y cuando recibe, está obligado a dar (aunque esa compensación pueda demorar meses y aún años); ese código es tan importante en el juicio xavante que el único que en esa comunidad no tiene derecho a casarse es el egoísta (XPA 60-71). Un mito deja entrever que el robo de una pieza de caza flechada por otro grupo (violación del intercambio) es causa de guerra (XX).

La tierra se distribuye para uso colectivo de los habitantes de una misma cabaña (hogar de varias familias que reciben según sus necesidades el uso-responsabilidad de un lote), y hay rotación de las áreas cultivadas al cabo de cinco o seis años, debido al agotamiento de la tierra, en medida de alto valor ecológico de la que los "no primitivos" blancos deberíamos mucho aprender (XPA, 82-83 y 224-230).

En esa comunidad altamente cohesionada por los lazos de solidaridad, no existe la figura del "niño abandonado" (tan común en las urbes de América Latina y del tercer mundo), pues la estructura de parentesco dentro de cada clan siempre prevé un responsable por el menor.

\subsubsection{Convergencias}

Las manifestaciones solidarias y ecológicas que acabamos de citar son modelos vivos para nuestra apuesta ecomunitarista.

\subsubsection{Diferencias}

La sociedad xavante vivió una cultura guerrera incompatible con las aspiraciones ecomunitaristas, aunque no falte el llamado a la clemencia con los vencidos (XX), empañado por la justificación de la esclavitud por motivos de guerra (XII). Además, y ya lo hemos notado en el Popol Vuh (y el hecho se repite en las diversas culturas indígenas amerindias), la libertad individual no ha sido puesta en la cultura xavante con la intensidad e 
importancia central que le confiere nuestro pensamiento de la liberación; de ahí que, por ejemplo, no admitamos como modelo la subordinación sistemática de los más jóvenes a los viejos y las tradiciones, y el papel rígidamente diferenciado de hombres y mujeres.

\subsubsection{La cultura xavante y la educación ambiental ecomunitarista (EAE)}

La EAE debe rescatar el gran amor de los xavantes por los niños, que se extiende explícitamente a los que nacen con deficiencias físicas o mentales (XPA, 141) y se revela en la autoflagelación de la madre que pierde un niño con menos de 7 años de edad (XPA, 266). Ese amor se prolonga en la atención y paciencia con la que los más viejos instruyen a los más jóvenes (lo que se corresponde con el respeto de éstos hacia aquellos).

También puede inspirarse la erótica ecomunitarista de la libertad sexual confesada incluso en un mito (XI), y en la sorprendente tolerancia con la que en un mito es tratado un joven sorprendido en incesto con su tía (X, simplemente se le perforó las orejas antes de la edad).

Y, como hemos dicho, la EAE debe educar en y para una economía solidaria y ecológica que se inspire del comportamiento xavante (aunque incorpore, como lo quiere el ecomunitarismo, los avances de la tecnología limpia).

\section{El ecomunitarismo y el Popol Vuh}

\subsection{El Popol Vuh}

El Popol Vuh es el libro de las tradiciones mitológicas e históricas de los indios quichés, pueblo asentado en Guatemala y cuyo centro de poder era la ciudad de Gumarcaah (Utatlán), sometida por los españoles en 1524 (que luego la rebautizaron con el nombre de Santa Cruz). La versión que nos ha llegado dice (al principio y al final del texto) ser una paráfrasis de un libro originario (el Popol Vuh) que se habría perdido con la conquista española; esta versión habría sido escrita por un sacerdote indígena bautizado con el nombre de Diego Reynoso, que la elaboró en caracteres latinos, fonetizando su lengua maya. El dominico español (que vivió entre 1666 y 1729), encargado de la evangelización en la región maya, realizó la primera traducción literal de la obra, que fue publicada recién en 1929 por la Sociedad de Geografía e Historia de Guatemala. Es curioso que antes lo hubiera sido en Viena en 1857 y en Paris en 1861. En 1947 Adrián Recinos publicó en Méjico la mejor versión académica del Popol Vuh y Agustín Estrada Monroy editó la versión paleográfica del texto quiché y su traducción española. 
La obra quiché no estaba dividida en partes, y las mismas varían según las ediciones. Pero la secuencia de los sucesos que narra sigue en su Primera Parte el siguiente orden: 1) Relato de la creación: donde se narra la obra de los dioses a partir del Universo vacío y la creación (frustrada en tres ensayos) de criaturas que los adoraran (los animales y los hombres de barro y de madera); 2) Relato de los tres soberbios: personajes castigados por su vanidad, y donde se incluye la historia de los Cuatrocientos Muchachos y el nacimiento y la infancia de los semidioses; 3) Relato de los dos hermanos semidioses Hunahpú y del submundo de la muerte; 4) Relato de la doncella Ixquic: incluye cuestiones sobre la femeneidad, el árbol prohibido y la concepción virginal; 5) Relato de Hunahpú e Ixbalanqué, que incluye el descubrimiento del juego de pelota; 6 ) Relato de los señores de la muerte; 7) Relato de las pruebas de Xibalbá: que incluye la resurrección de Hunahpú; 8 ) Relato del triunfo de los héroes: incluye el nacimiento del Sol y de la Luna y la transformación de los Cuatrocientos Muchachos en estrellas, y, 9) Relato de la creación del hombre actual (a partir del maíz) y la creación de las mujeres. La Segunda Parte contiene: 10) El legado de los dioses: historia del pueblo quiché, con la multiplicación de los humanos y la diversificación de los pueblos mayas y sus lenguas, y la adquisición del fuego; 11) Culminación de la obra de la creación, con el amanecer; 12) Relato de la guerra de las tribus: con la victoria quiché; 13) Relato de la muerte de los varones, el viaje hacia oriente y las sucesiones dinásticas; 14) Relato de la grandeza quiché: glorificación de su imperio, con el aumento de su capital donde en diversos templos se cumplen las ofrendas y los ayunos rituales; y 15) Relato de la genealogía de los señores quichés, que incluye al primer Conquistador español (Pedro de Alvarado, llamado Donadiú, deformación de 'Tonatiuh', o sea, el sol, en, náhuatl).

A continuación haremos uso de esa numeración para localizar los temas en la obra.

\subsection{Ecomunitarismo y Popol Vuh: revelaciones, convergencias y diferencias}

\subsubsection{Revelaciones}

Como sucede con los mitos, los relatos contienen "explicaciones" para el origen de ciertos seres o costumbres; así, por ejemplo, se explica la creación de ciertos animales y de la humanidad (1 y 9), de los monos (5, hombres transformados), de las diversas tribus mayas y su diversidad lingüística $(9,10$ y 11) y su demografía (12) y de las "grandes casas nobles" e imperio (14) y de la esclavitud (12), origen del Sol, la Luna y las estrellas (2, 8 y 11), de los frutos del jícaro (3); y también se explica el origen de ciertas costumbres y características: origen de los sacrificios humanos masivos y del ayuno ritual (10 y 13), de las fiestas de casamiento (entre nobles, 13), de por qué hay animales carnívoros (1), de por qué el mos- 
quito chupa sangre, de por qué los ratones tienen la cola pelada $(5,6)$, de por qué el mochuelo tiene la boca partida (7) ; e incluso se explica cuáles semidioses deciden de ciertas enfermedades (3).

Llaman la atención ciertas semejanzas con mitos bíblicos, como es el caso del intento de creación (fallido) de los hombres a partir del barro (1, como en el Génesis), la incomunicación de las tribus a causa de la diversificación de su lengua a partir de un tronco inicialmente común (9, como en la torre de Babel), el diluvio (10, como el del arca de Noé) y su fin (11), la concepción de una virgen (4, como María), la resurrección de un héroe (8, como la de Jesús) e incluso de ciertos animales (6). Y también hay una semejanza y una diferencia en relación al mito prometeico griego sobre cómo los seres humanos se apropiaron del fuego, pues si a instancias de lo que ocurre en el mito griego los quiché recibieron inicialmente el fuego de manos de los dioses, luego una tribu aprendió a fabricarlo a partir del frotamiento de dos maderas (10, con lo que la obra asume contornos etnográficos e incluso tecnológicos).

\subsubsection{Convergencias}

3.2.1.1. Edad de oro - Aunque como en otros mitos y tradiciones históricas la situación de convivencia harmoniosa y pacífica se pone en un pasado remoto, no podemos sino reivindicar desde el ecomunitarismo la celebración que se hace en el Popol Vuh de un reino en el que las gentes "se mantuvieron unidas, sin discordias ni rencillas, y el reino vivía en calma y no había guerras ni revueltas; sólo la paz y la felicidad reinaban en sus corazones" (13).

3.2.1.1.2. Gobierno rotativo - Aunque la alternancia se da entre señores de una aristocracia, no podemos dejar de saludar desde la "política de todos" ecomunitarista, un gobierno rotativo, donde "de dos entraban [en sus funciones] y así se sucedían para encargarse del pueblo y de todos los hombres del Quiché" (14)

3.2.1.1.3. Trabajo diversificado y comida - La obra, indirectamente, celebra la diversidad de la actividad creadora como siendo buena para el desarrollo individual (en conformidad con la utopía marxiana y ecomunitarista); así un héroe educó a sus dos hijos de tal modo "que llegaron a ser músicos, cantores, tiradores de cerbatana, pintores, escultores, talladores y joyeros" (3). Y el Popol Vuh deja claro, aunque incidentalmente y de forma negativa, que el deber incluido en el principio ecomunitarista "de cada uno según su capacidad y a cada uno según su necesidad" es el de producir para la comunidad de la que se recibe lo necesario para el libre y multilateral desarrollo individual; en efecto allí se lee: "Comieron seguidamente, pero en verdad no habían trabajado en el campo, de modo que recibieron su comida sin merecerla" (5). 


\subsubsection{Diferencias: la dificultad del diálogo intercultural}

Hace varios años y dialogando con los colegas que en América Latina quieren hacer una filosofía intercultural, llamé la atención para la dificultad básica de dialogar con culturas (como las indígenas de A. Latina) donde parece que el lenguaje no tiene el uso argumentativo que heredamos de Grecia (que permite el debate sobre la verdad, incluso en los enunciados que sustentan en mi ética a los obligativos constitutivos de las normas éticas) y la individualidad (y por ende el concepto de "liberación") no tiene el estatus que le conferimos desde la modernidad capitalista, y, en especial, en el pensamiento de la liberación (no en vano, tanto Hegel como Marx, nos hablaron del "individuo universal" como la meta de la instauración del reino de la libertad; no otra cosa digo desde mi propuesta ecomunitarista).

Una vez que se constata esta situación, dije que debíamos aprender con esas culturas (en especial en la temática ecológica, donde desde ya podemos luchar juntos, así como en el combate a la opresión invasora del capitalismo globalizado) y evitar su destrucción por la nuestra, esperando que un futuro incierto quizá el diálogo se haga posible; hasta ese momento, respetuosamente sólo les podemos sugerir nuestra discrepancia con la condena del saber (Popol Vuh, 9, semejante a la condena a Adán y Eva), la posición secundaria que la mujer ocupa en muchas de esas culturas (instrumentos sexuales en el Popol Vuh, 12, aunque las hay guerreras, como en 12, también), la organización jerárquica e imperial de la sociedad, y otros asuntos de primera importancia.

3.2.1.3 Algunas aplicaciones del Popol Vuh a la educación ambiental ecomunitarista (EAE)

\subsection{La Naturaleza no humana es amiga y/pero se venga de los humanos} si es maltratada

En una época en la que cada vez queda más clara nuestra inserción como una especie más del vasto ecosistema planetario y la tercera norma de la ética nos exige mantener los grandes equilibrios ecológicos, preservando y regenerando una naturaleza sana, mucho podemos aprender de la manera como el Popol Vuh presenta a seres no humanos que son amistosos y serviciales con humanos (un piojo mensajero, en 6, hormigas que hacen el trabajo de dos jóvenes, en 7), pero también nos refiere el castigo que infligen a los humanos que no los respetan; así, hombres que olvidaron al "Espíritu del Cielo, su madre y su padre", son recriminados y castigados por "animales pequeños y grandes", "palos y piedras que golpearon los rostros", "tinajas, platos, ollas, perros, y piedras de moler" (1); y parecido castigo sufren ciertos señores soberbios de abejas y avispas (12). 


\subsection{Castigo a los soberbios y/o malvados}

Si el Popol Vuh condena a los insensibles con la naturaleza no humana, también y varias veces (y especialmente en 2 ) condena con el castigo a los que son malvados o soberbios con sus congéneres. En una EAE que abarque las esferas familiar, institucionales y comunitarias, tiene lugar destacado (en consonancia con la segunda norma de la ética) la prédica con el ejemplo y la palabra del respeto hacia las opiniones y vidas ajenas.

\subsubsection{Dieta: ayunos y abstinencia sexual}

La EAE no puede obviar la dimensión dietética y sexual; el Popol Vuh celebra los ayunos y la abstinencia sexual; Gandhi adoptó una y otra práctica (la segunda antes de los 40 años de edad). Desde la EAE y la frugalidad que ella defiende, apoyamos a los ayunos y no suscribimos la abstinencia sexual, pues no vemos (a no ser que haya de por medio experiencias negativas, o enfermedades) en qué tal abstinencia puede favorecer el equilibrio físico y mental del individuo que queremos libre y multilateralmente (por lo tanto, también sexualmente) desarrollado.

\section{Bibliografía}

Giaccaria, Bartolomeu \& Heide, Adalberto (1975). Jerônimo xavante conta, Ed. Casa da Cultura, Campo Grande.

Giaccaria, Bartolomeu \& Heide, Adalberto (1984), Xavante, povo autêntico, Ed. Salesiana Dom Bosco, São Paulo.

Lopez Velasco, Sirio (2003a). Fundamentos lógico-lingüísticos da ética argumentativa, Ed. Nova Harmonia, São Leopoldo (Brasil).

Lopez Velasco, Sirio (2003b). Ética para o século XXI: rumo ao ecomunitarismo, Ed. Unisinos, São Leopoldo (Brasil).

López Velasco, Sirio (2003c). Ética para mis hijos y no iniciados, Ed. Anthropos, Barcelona (España).

López Velasco, Sirio (2007). Alias Roberto: diario ideológico de una generación, Ed. Baltgráfica, Montevideo (Uruguay).

Lopez Velasco, Sirio (2008). Introdução à educação ambiental ecomunitarista, Ed. FURG, Rio Grande (Brasil).

Popol Vuh, edición organizada por Manuel A. Canseco, Ed Nuevo Mundo, Montevideo, 1987. 